\title{
PRENATAL DYSTHYMIA VERSUS MAJOR DEPRESSION EFFECTS ON MATERNAL CORTISOL AND FETAL GROWTH
}

Tiffany Field, Ph.D., ${ }^{1,2 *}$ Miguel A. Diego, Ph.D., ${ }^{1}$ Maria Hernandez-Reif, Ph.D., ${ }^{1}$ Barbara Figueiredo, Ph.D., ${ }^{3}$ Angela Ascencio, ${ }^{1}$ Saul Schanberg, M.D., Ph.D., ${ }^{4}$ and Cynthia Kuhn, Ph.D. ${ }^{4}$

To determine differences between pregnant women diagnosed with Dysthymia versus Major Depression, depressed pregnant women $(N=102)$ were divided by their diagnosis into Dysthymic $(N=48)$ and Major Depression $(N=54)$ groups and compared on self-report measures (depression, anxiety, anger, daily bassles and behavioral inhibition), on stress hormone levels (cortisol and norepinephrine), and on fetal measurements. The Major Depression group had more self-reported symptoms. However, the Dysthymic group had higher prenatal cortisol levels and lower fetal growth measurements (estimated weight, femur length, abdominal circumference) as measured at their first ultrasound ( $M=18$ weeks gestation). Thus, depressed pregnant women with Dysthymia and Major Depression appeared to have different prenatal symptoms. Depression and Anxiety 25:E11-E16, 2008. Published 2008 Wiley-Liss, Inc.

Key words: dysthymia; MDD; cortisol; fetal growth

\section{INTRODUCTION}

Many researchers studying the effects of maternal
depression on prenatal development have grouped
depression diagnoses, namely Major Depression Dis-
order and dysthymia mothers together. These dis-
orders may have very different effects on prenatal
development if the mothers are experiencing these
disorders differently during pregnancy. Thus, the
purpose of this study was to explore the differences
between pregnant women experiencing Major Depres-
sion Disorder (MDD) and Dysthymia on: (1) their self-
reported feelings including depressive symptoms,
anxiety and anger, their daily hassles and their style of
coping (behavioral approach or withdrawal); (2) their
neuroendocrine stress hormones (cortisol and norepi-
nephrine); and (3) the growth measures of the fetuses
from ultrasounds.
Major depression is usually considered more severe
than dysthymia. However, the literature is somewhat
mixed on that issue. For example, major depression has
been considered by some researchers to be more severe
and less chronic than Dysthymia [Klonsky and
Bartelson, 2000]. In the study by Klonsky and
Bartelson [2000], more physical/somatic symptoms
were noted for Major Depression than Dysthymia,
${ }^{1}$ Touch Research Institutes, University of Miami School of Medicine, Miami, Florida

${ }^{2}$ Department of Psychology, Fielding Graduate University, Santa Barbara, California

${ }^{3}$ Department of Psychology, University of Minho, Braga, Portugal

${ }^{4}$ Department of Pharmacology, Duke University Medical School, Durham, North Carolina

Contract grant sponsor: Senior Research Scientist Awards; Contract grant number: MH 00331 and AT 001585; Contract grant sponsor: NIMH merit award; Contract grant number: $\mathrm{MH}$ 46586; Contract grant sponsor: NIH grant; Contract grant number: AT 00370; Contract grant sponsor: March of Dimes grant; Contract grant number: 12-FY03-48.

*Correspondence to: Dr. Tiffany Field, Touch Research Institutes, University of Miami Medical School, Department of Pediatrics (D-820), P.O. Box 016820, Miami, FL 33101.

E-mail: tfield@med.miami.edu

Received for publication 17 May 2006; Revised 10 January 2007; Accepted 30 January 2007

DOI 10.1002/da.20307

Published online 22 June 2007 in Wiley InterScience (www. interscience.wiley.com).

†This article is a US Government work and, as such, as in the public domain in the United States of America. 
leading to the authors' suggestion that Major Depressive Disorder is more severe than Dysthymia. In another study addressing the psychosocial correlates and treatment seeking of these two groups, the affective symptoms appeared to be similar for Major Depression and Dysthymia [Flament et al., 2001]. However, the Major Depression group had more comorbid conditions including anxiety disorders, suicidal behaviors and alcohol intoxication, as well as more severe stressors at the onset of their illness. Other factors such as peer relationships and pathological behaviors did not differ between the groups, although treatment seeking was greater in the Major Depression group (35\%) than it was in the Dysthymic group (24\%).

Others have suggested that although Dysthymia is a chronic, low grade form of depression, its morbidity rate is as high as that of Major Depression, and Dysthymia would be expected to increase the risk for Major Depressive Disorder [Griffiths et al., 2000]. These authors suggested that Dysthymia may stem from disturbances of neuroendocrine and neurotransmitter functioning (e.g. Corticotropin Releasing Hormone, the precursor of cortisol, or norepinephrine). They also claimed that the persistence of the syndrome makes those individuals also vulnerable to major depression. Neurochemical disturbances associated with Dysthymia may also serve to increase the recurrence or relapse rate. In addition, lower ratings for social functioning have been reported for dysthymia [Yang and Dunner, 2001].

In other studies, groups with Major Depression and Dysthymia could not be differentiated on sociodemographic, clinical, family or life events variables [Goodman et al., 2000; McCullough et al., 2000]. Thus, this literature is limited and inconclusive. Further, no literature could be found on the differential effects of these different types of depression on pregnancy.

This study is the first to explore the differential effects of Major Depression and Dysthymia on pregnant women's psychological stress, on their cortisol and norepinephrine levels, and on the measurements of their fetuses in the same study. Prenatal cortisol and norepinephrine were assayed inasmuch as Griffiths et al. [2000] had suggested that cortisol and norepinephrine may be elevated in these conditions. Also, cortisol has been implicated as a contributor to prematurity [Field et al., 2004] and norepinephrine has been correlated with uterine artery resistance and fetal growth deprivation via decreased blood flow to the fetus [Glover et al., 1999].

\section{METHOD}

\section{PARTICIPANTS}

The participants were 102 depressed pregnant women $(N=48$ with Dysthymia, $N=54$ with Major
Depression). The mothers averaged 27.2 years of age, their socioeconomic status was low to middle $(M=4.0$ on the Hollingshead Index), and they were distributed 54\% Hispanic, 26\% African American and 20\% nonHispanic White.

\section{PROCEDURE}

Two hundred-eighty pregnant women were recruited at prenatal clinics at their first ultrasound visit $(M=18$ weeks gestation, $R=16-22$ weeks). Following informed consent, the 280 pregnant women were administered the Structured Clinical Interview for Depression (SCID) by research associates supervised by a clinical psychologist. Although illicit drug use was an exclusionary criterion, none of the women reported this problem. Of the sample recruited, 102 of the women were diagnosed with Dysthymia $(N=48)$ or Major Depression $(N=52)$ based on the SCID. The two groups of women did not differ on their gestational age at the time of their ultrasounds. Women who met the criteria for both MDD and Dysthymia were excluded $(N=14)$ given their relatively smaller sample size. Similarly, co-morbid depression and anxiety women were excluded because they were unevenly distributed across the MDD and Dysthymia groups.

The depressed pregnant women were then given selfreport measures including the Center for Epidemiological Studies-Depression (CES-D) scale for depressive symptoms, the State/Trait Anxiety Inventory (STAI), the State/Trait Anger Inventory (STAXI), the Daily Hassles scale, and the Behavioral Inhibition and Activation scales (BIS/BAS). The ultrasonographer recorded fetal measurements including biparietal diameter, femur length, head circumference, and abdominal circumference, and from those measures, fetal weight was calculated. Finally, the mothers provided a morning urine sample to be assayed for prenatal cortisol and norepinephrine levels.

\section{STRUCTURED CLINICAL INTERVIEW FOR DSM-IV AXIS I DISORDERS (SCID-D)}

All women in the study were given the SCID-I (nonpatient edition: research version) interview to determine depression and anxiety diagnoses and to screen out other disorders including bipolar disorder, schizophrenia and other psychotic disorders. The women were diagnosed with Dysthymia or Major Depression on the SCID based on DSM IV symptoms. The SCID was administered by research associates following training and with continuing supervision by a clinical psychologist. These depressed women were also referred to our collaborating psychiatrist at the medical school psychiatric treatment center.

Center for Epidemiological Studies Depression Scale. This 20 -item scale was included to assess symptoms of depression [Radloff, 1977]. The subject is asked to report on her feelings during the preceding week. The scale has adequate test/retest reliability 
(.60 over several weeks), internal consistency (.80-.90) and concurrent validity [Wells et al., 1987]. Scores can range from 0 to 60 . A score of 16 on the CES-D is considered the cut-point for depression [Radloff, 1991].

\section{SELF-REPORT MEASURES}

State/Trait Anxiety Inventory. This scale was used to assess anxiety levels in the different depression groups [Spielberger et al., 1970]. The State/Trait Anxiety Inventory is composed of 20 items and is summarized by a score ranging from 20 to 90 and assesses how the subject usually feels in terms of severity ("not at all" to "very much so"). Characteristic items include "I feel nervous" and "I feel calm." Research has demonstrated that the STAI has adequate concurrent validity and internal consistency $(r=.83)$. The cutoff score for high anxiety is 48 .

State/Trait Anger Inventory. This is a 10 -item inventory that assesses general feelings of anger based on a four-point Likert scale ranging from 1 (almost never) to 4 (almost always) [Spielberger et al., 1983]. Typical questions include "I am quick tempered" and "I fly off the handle". Scores can range from 10 to 40 . Psychometric properties have been established for the STAXI on diverse ethnic groups. Reliability coefficients have been reported between 0.97 (state) and 0.89 (trait).

Daily Hassles Scale. This scale was developed to assess the degree of hassle being experienced by expectant mothers [Field, 2003]. The 16 items on a four-point Likert scale include questions on people such as family members, landlord and friends creating hassles and the act of finding resources as creating hassles. Scores can range from 16 to 64 .

The Behavioral Inhibition/Behavioral Approach System Questionnaire. The BIS/BAS is a 24-item questionnaire consisting of personal statements followed by four severity options ranging from very true to very false [Carver and White, 1994]. Scores can range from 24 to 96 . The BIS/BAS is designed to assess the tendency to behave in response to reward or in response to punishment. High BIS scores reflect withdrawal behavior, and high BAS scores reflect intrusive behavior.

Urine assay. Urines were collected in specimen containers by the women during their morning ultrasound clinic visits. Although several saliva samples may have been more representative of $24 \mathrm{hr}$ Hypothalamic Pituitary Adrenal axis function, the urines were a convenience sample because the mothers' time at the clinic was limited, and urine samples were routine for clinic. They were then frozen, and sent to Saul Schanberg, M.D., Ph.D. at Duke University Medical School, where they were assayed for cortisol and norepinephrine. Cortisol was measured in urine by radioimmunoassay using a specific antiserum from Radioassay Systems Laboratories. The sensitivity of the assay is $0.025 \mathrm{ng} /$ tube. The inter-assay and intra-assay coefficient of variation is less than $10 \%$ and $5 \%$, respectively. Analysis of urinary norepinephrine was conducted by high-pressure liquid chromatography (HPLC) and electrochemical detection.

Ultrasound assessments. These assessments were conducted at 16-22 weeks gestation at the university ultrasound clinic and were the only scheduled ultrasound appointments for these women. All ultrasound measurements were performed by clinical ultrasonographers using a color ultrasound machine with 3.5 and 5-Mhz curvilinear abdominal probes. All ultrasonographers were blind to the diagnoses of the pregnant women.

Fetal measurements. Fetal measurements including femur length (FL), abdominal circumference (AC), head circumference (HC) and biparietal diameter (BPD) were made in centimeters using standard clinical measurement protocols [Chitty et al., 1994]. The Shepard et al. [1982], fetal weight estimation algorithms were used to estimate fetal weight from fetal ultrasound measurements.

\section{RESULTS}

Multivariate analyses of variance (MANOVAs) were conducted on the self-report measures, the biochemical measures, and fetal growth measures. Following significant MANOVAs, univariate ANOVAs were conducted with the group assignment (Dysthymia/Major Depression) being the between groups measure. On the demographic variables, the groups differed on socioeconomic status $(M=4.2$ for the Dysthymic group and $M=3.8$ for the Major Depression group, $F=4.09$, $P<.05)$. Inasmuch as the groups differed on this background measure, socioeconomic status was entered as a covariate in the analyses.

\section{SELF-REPORT MEASURES}

As can be seen in Table 1, the Major Depression group received more severe scores on all of the selfreport measures. Their higher scores suggested: (1) more depressive symptoms on the CES-D; (2) higher anxiety levels on the STAI; (3) higher anger levels on the STAXI; (4) more hassles on the Daily Hassles scale; and (5) greater inhibition on the BIS/BAS scales.

\section{BIOCHEMICAL MEASUREMENTS}

As can be seen in Table 2, the Dysthymic group had higher prenatal cortisol levels, although their norepinephrine levels did not differ from those of the Major Depression group.

\section{FETAL GROWTH MEASURES}

As can be seen in Table 2, the Dysthymic group had lower fetal measurements including femur length, abdominal circumference, and fetal weight. 
TABLE 1. Pregnancy self-report measures (standard deviations in parentheses)

\begin{tabular}{lccc}
\hline & \multicolumn{2}{c}{ Groups } & \\
\cline { 2 - 4 } Measures & Dysthymic & Major depression & $F$ \\
\hline Depression (CES-D) & $23.4(9.5)$ & $30.7(9.1)$ & 15.27 \\
Depressed affect & $6.6(3.3)$ & $7.3(4.4)$ & .000 \\
Somatic/vegetative & $6.9(3.3)$ & $9.9(2.7)$ & NS \\
Positive affect & $5.0(3.2)$ & $6.8(1.7)$ & .003 \\
Interpersonal distress & $1.2(1.3)$ & $1.8(1.0)$ & .05 \\
Anxiety (STAI) & $45.2(10.4)$ & $49.8(10.4)$ & NS \\
Anger (STAXI) & $18.4(4.5)$ & $22.2(6.2)$ & .03 \\
Daily hassles & $24.7(7.5)$ & $28.2(7.7)$ & .00 \\
Behavioral inhibition & $16.1(2.7)$ & $19.3(3.6)$ & .003 \\
Behavioral activation & $38.1(8.4)$ & $41.3(7.2)$ & .05 \\
\hline
\end{tabular}

TABLE 2. Maternal biochemical and fetal growth data (standard deviations in parentheses)

\begin{tabular}{|c|c|c|c|c|}
\hline \multirow[b]{2}{*}{ Measures } & \multicolumn{2}{|c|}{ Groups } & \multirow[b]{2}{*}{$F$} & \multirow[b]{2}{*}{$P$} \\
\hline & Dysthymic & Major depression & & \\
\hline \multicolumn{5}{|l|}{ Biochemical } \\
\hline Prenatal cortisol & $382.8(256.6)$ & $213.4(124.6)$ & 3.86 & .05 \\
\hline Prenatal norepinephrine & $63.8(15.0)$ & $63.0(16.8)$ & & NS \\
\hline \multicolumn{5}{|l|}{ Fetal growth } \\
\hline Fetal weight $\mathrm{a}^{\mathrm{a}}$ & $446.3(348.5)$ & $485.9(344.6)$ & 3.42 & .05 \\
\hline Biparietal diameter ${ }^{\mathrm{b}}$ & $4.9(2.2)$ & $6.8(4.9)$ & & NS \\
\hline Femur length ${ }^{\mathrm{b}}$ & $3.5(2.2)$ & $4.8(3.1)$ & 3.37 & .05 \\
\hline Head circumference ${ }^{b}$ & $15.7(6.1)$ & $24.0(19.5)$ & & NS \\
\hline Abdominal circumference ${ }^{\mathrm{b}}$ & $14.0(5.9)$ & $23.4(14.6)$ & 3.38 & .05 \\
\hline
\end{tabular}

${ }^{\mathrm{a}}$ Grams.

${ }^{\mathrm{b}}$ Centimeters.

\section{DISCUSSION}

The more severe scores on the self-report measures of the Major Depression group suggested greater symptoms for that group. This group scored higher on the depressive symptoms scale (CES-D) as well as the somatic/vegetative and positive affect subscales of the CES-D. They also received higher scores on the anxiety (STAI), anger (STAXI), inhibition (BIS/BAS) and Daily Hassles measures. The greater symptoms for the Major Depression group in this study are consistent with those noted by Klonsky and Bartelson [2000], who reported that their Major Depression group had more severe physical/somatic symptoms. This self-report profile is also consistent with more comorbid conditions associated with Major Depressive Disorder, as opposed to Dysthymia, including anxiety disorder [Flament et al., 2001]. The higher inhibition scores for the Major Depression group may be consistent with their higher anxiety scores. Although daily hassles are often associated with lower socioeconomic status, the
Major Depression group was reporting more daily hassles, even though their socioeconomic status was higher than that of the Dysthymic group. The reporting of more symptoms on all of these scales by the Major Depression group may relate to the acute nature of their depressive episodes. The Dysthymic group may be under-reporting symptoms that they have grown accustomed to by virtue of their chronic condition.

The inconsistency between the self-report measures and the maternal biochemical and fetal measurements data are somewhat surprising. Although the Major Depression group was scoring worse on the self-report measures, the Dysthymic group had significantly higher prenatal cortisol levels, which may have contributed to the lower fetal weight, lesser femur length and lesser abdominal circumference noted in the ultrasound examinations of their fetuses. In previous studies [Field et al., 2004], elevated cortisol has been associated with lower birthweight. Typically, prenatal norepinephrine is also inversely related to fetal 
measurements [Glover et al., 1999]. However, in this study, the groups were comparable on prenatal norepinephrine. The elevated cortisol and lower fetal measurements appear to be a consistent profile, and one that has been described by others as disturbed neuroendocrine and neurotransmitter functioning [Griffiths et al., 2000]. We have noted significantly higher cortisol levels and lower fetal growth measures in depressed women versus non-depressed women and their fetuses [Diego et al., 2006], and the cortisol levels are higher and fetal growth measures lower in these Dysthymia and MDD groups than in the Diego et al. [2006] study. These neurochemical imbalances may contribute to a Dysthymic state which may, in turn, affect fetal measurements. The chronicity of this state may contribute to the Dysthymic women underreporting their symptoms or even experiencing fewer symptoms than are typical of Dysthymia.

Thus, a dichotomous picture emerged, with the Major Depression group appearing to have more severe symptoms based on self-report measures, and the Dysthymic group appearing to have more imbalanced prenatal biochemistry and smaller fetal measurements. None of these differences are likely to be treatment effects (which generally favor the Major Depression group), because none of these women were receiving treatment during pregnancy. Further research is needed on other self-report measures and biochemical and physiological variables and across pregnancy to determine the validity of these different profiles. The lack of prenatal treatment (both psychotherapy and antidepressant medications) in these groups, whereas not surprising given earlier studies documenting the under-treatment of prenatal depression, highlights the need for this at-risk population to be recognized and referred for treatment more readily.

Both the Major Depression and Dysthymic groups would, then, appear to have prenatal problems. The Major Depression group, based on the self-reported symptoms (depression, anxiety, anger, inhibition and daily hassles), appear to be aware of their distress. Although this is not reflected in their prenatal cortisol levels, their felt distress may be affecting other chemistry that was not measured here. In contrast, the Dysthymic group had a less severe pattern of selfreport problems, although they seemed to be distressed, as manifested by their significantly higher cortisol levels, and by the effects cortisol may have had on their inferior fetal measurements including fetal weight, femur length and abdominal circumference. The other measurements (biparietal diameter and head circumference), although not significantly lower, tended to be lower in the Dysthymic group fetuses. Although the literature suggests that Major Depression Disorder may be the more severe diagnosis [Klonsky and Bartelson, 2000], these data highlight a potential biochemical imbalance and lower fetal measurements for the Dysthymic group, suggesting that both types of measures on both groups are in need of further study.

In any future study, the limitations of this study need to be corrected. The inclusion of a control group makes it easier to interpret the extent of risk for these depressed groups. Comparisons between women who had recurrent MDD versus single episode MDD would also be important. Checking reliability of the SCID examiners and the ultrasonographers would also be important. Nonetheless, these preliminary findings highlight the need for further studying pregnant women with MDD and Dysthymia.

Acknowledgments. We thank the mothers who participated in this study. This research was supported by Senior Research Scientist Awards (MH 00331 and AT 001585), an NIMH Merit Award (MH 46586) an NIH grant (AT 00370) and March of Dimes grant (12-FY03-48) to Tiffany Field and funding from Johnson and Johnson Pediatric Institute.

\section{REFERENCES}

Carver CS, White TL. 1994. Behavioral inhibition, behavioral activation, and affective responses to impending reward and punishment: the BIS/BAS scales. J Pers Social Psychol 67: 319-333.

Chitty LS, Altman DG, Henderson A, Campbell S. 1994. Charts of fetal size: 2 head measurements. Br J Obstet Gynecol 101: $35-43$.

Diego M, Jones N, Field T, Hernandez-Reif M, Schanberg S, Kuhn C, Gonzalez-Garcia A. 2006. Maternal Psychological Distress, prenatal cortisol and fetal weight. Psychosomatic Med 68:747-753.

Field T. 2003. Daily Hassles Scale, unpublished scale.

Field T, Diego M, Hernandez-Reif M, Vera Y, Gil K, Schanberg S, Kuhn C, Gonzalez-Garcia A. 2004. Prenatal maternal biochemistry predicts neonatal biochemistry. Int J Neurosci 114: 933-945.

Flament MF, Cohen D, Choquet M, Jeammet P, Ledoux S. 2001. Phenomenology, psychosocial correlates, and treatment seeking in major depression and dysthymia of adolescence. J Am Acad Child Adolesc Psychiatry 40:1070-1078.

Glover V, Teixeira J, Gitau R, Fisk NM. 1999. Mechanisms by which maternal mood in pregnancy may affect the fetus. Contemporary Rev Obstetr Gynecol 1-6.

Goodman SH, Schwab-Stone M, Lahey B, Shaffer D, Jensen PS. 2000. Major depression and dysthymia in children and adolescents: discriminant validity and differential consequences in a community sample. J Am Acad Child Adolesc Psychiatry 39:761-770.

Griffiths J, Ravindran AV, Merali Z, Anisman H. 2000. Dysthymia: a review of pharmacological and behavioral factors. Mol Psychol 5:242-261.

Klonsky ED, Bartelson AD. 2000. MMPI-2 clinical scale differences between dysthymia and major depression. Assessment 7: 143-149.

McCullough JP Jr, Klein DN, Keller MB, Holzer CE III, Davis SM, Kornstein SG, Howland RH, Thase ME, Harrison WM. 2000. Comparison of DSM-III-R chronic major depression and major depression superimposed on dysthymia (double depression): validity of the distinction. J Abnorm Psychol 109:419-427.

Radloff L. 1977. The CES-D scale: a self-report depression scale for research in the general population. Appl Psychol Methods 1:385-401. 
Radloff L. 1991. The use of the Center for Epidemiological Studies Depression, Scale in adolescents and young adults. J Youth Adolesc 20:149-165.

Shepard MJ, Richards VA, Berkowitz RI. 1982. An evaluation of two equations for predicting fetal weight by ultrasound. Am J Obstet Gynecol 147:47.

Spielberger CD, Gorsuch TC, Lushene RE. 1970. The State/Trait Anxiety Inventory. Palo Alto, CA: Consulting Psychologists Press.
Spielberger CD, Jacobs G, Russell S, Crane R. 1983. Assessment of anger: the State/Trait Anger Scale. In: Butcher JN, Spielberger $\mathrm{CD}$, editors. Advances in personality assessment, 2. Hillsdale, NJ: Erlbaum. p 159-187.

Wells VE, Klerman GL, Deykin EY. 1987. The prevalence of depressive symptoms in college students. Social Psychiatry 22: 20-28.

Yang T, Dunner DL. 2001. Differential subtyping of depression. Depression Anxiety 13:11-17. 